\title{
Quintessence, Cosmological Horizons, and Self-Tuning
}

\author{
by J.M. Cline \\ McGill University, 3600 University St., Montréal, Qc H3A 2T8, Canada \\ E-mail: jcline@physics.mcgill.ca
}

\begin{abstract}
We point out that quintessence with an exponential potential $V_{0} e^{-\beta Q / \sqrt{3} M_{p}}$ can account for the present observed acceleration of the universe, without necessarily leading to eternal acceleration. This occurs for $2.4<\beta<2.8$. Thus a cosmological horizon, which is supposed to be problematic within the context of string theory, can be avoided. We argue that this class of models is not particularly fine-tuned. We further examine this question in the context of a modified Friedmann equation, $H^{2} \propto \rho+p$, which is suggested by higher dimensional self-tuning approaches to the cosmological constant problem. It is shown that the self-tuning case can also be consistent with observations, if $1.8<\beta<2.4$. Future observations of high- $z$ supernovae will be able to test whether $\beta$ lies in the desired range.
\end{abstract}




\section{Contents}

1. Introduction 1

2. Quintessence evolution and event horizon 3

3. Results 5

4. Naturalness 8

5. Conclusion 10

\section{Introduction}

There is convincing evidence that the universe is presently dominated by a form of dark energy density which is decreasing significantly more slowly with time than the energy density of ordinary matter. The first Doppler peak in the cosmic microwave background fluctuations is strongly consistent with a flat universe [1], whose density is the critical one $\rho_{c}$, yet the matter energy density is known by other means to be no more than $\Omega_{m}=\rho_{m} / \rho_{c} \sim 0.3$ [2]. Recent observations of the higher peaks by the various CMB experiments give $\Omega_{m}=0.25$ [3]. The Hubble diagram deduced from high redshift type I supernovae provides independent evidence for an additional component $\Omega_{\Lambda}$ of the energy density [4]. From an empirical viewpoint, pure vacuum energy $(\Lambda)$ is the simplest explanation, but theoretically it is difficult to explain why $\Lambda$ is some 124 orders of magnitude smaller than the natural scale set by the Planck mass, $M_{p}^{4}$. If the dark energy is due to a rolling scalar field $Q$, quintessence [5], whose potential energy vanishes as $Q \rightarrow \infty$, this might be a more natural explanation for why the dark energy density is small.

A further motivation for quintessence could be coming from string theory, because of its apparent incompatibility with de Sitter space [6]. An eternally accelerating universe, which would result from a positive cosmological constant, seems to be at odds with string theory, because of the impossibility of formulating the S-matrix. In de Sitter space the presence of an event horizon, signifying causally disconnected regions of space, implies the absence of asymptotic particle states which are needed to define transition amplitudes. Quintessence, on the other hand, would seem to offer the possibility of temporary acceleration to account for current observations, without necessarily making the scale factor of the universe accelerate forever. 
Recent papers have pointed out that, in fact, quintessence generically does lead to eternal acceleration, if the universe is accelerating now [0, 8] (see also [9]). Therefore it is concluded that string theory is equally at odds with quintessence or a positive cosmological constant. The purpose of the present paper is to explore some simple loopholes to this conclusion. ${ }^{1}$

Our initial motivation was an interesting possibility coming from the braneworld scenario, in particular, attempts to address the cosmological constant problem through self-tuning solutions to the Einstein equations [11]. In this approach, it is assumed that our universe is a 3-brane with arbitrary tension $\Lambda$, embedded in an extra dimension. A scalar field living in the extra dimension adjusts itself so as to yield a static solution to the Einstein equations, regardless of the value of $\Lambda$, which otherwise would act like the 4-D cosmological constant and lead to inflation of the brane. Although there are many problems with this idea [12], ref. [13] explored the question of how cosmology would be affected for brane observers assuming an acceptable model of self-tuning was found. If the scalar couples only to the volume element $\sqrt{g} d^{4} x$ of the three-brane, [13] showed that it is possible to obtain a modified Friedmann equation of the form

$$
H^{2}=\left(\frac{\dot{a}}{a}\right)^{2}=2 \pi G(\rho+p)
$$

plus corrections of order $G(\rho+p)^{2} / \mathrm{TeV}^{4}$ (assuming the 5 -D quantum gravity scale is of order $\mathrm{TeV}$ ). This is a very interesting twist on normal cosmology because (1) it leads to no expansion in the case of vacuum energy, where $p=-\rho$; (2) it is indistinguishable from the normal equation during the radiation dominated era, since $p=\rho / 3$; and (3) it gives a Hubble rate only $\sqrt{3 / 4}=0.87$ times smaller than normal during the matter dominated era, which would be difficult to distinguish from the standard value given the uncertainties on $\Omega_{m}$ (the fraction of the critical density in matter) and $H_{0}$ (the present value of the Hubble parameter). Since the modified Friedmann equation eliminates conventional inflation, it would remove the obstacle to defining an S-matrix in the 4-D universe when $\Lambda>0$. We might also expect it to ameliorate the problem with eternal acceleration in quintessence models.

Moreover, if the equation of state for the dark energy, $w=p / \rho$, turns out to be $w>-1$, as is still allowed by the supernova data, this could be indirect evidence for a modified Friedmann equation. Taking the time derivative of (1.1), one can show that the acceleration is

$$
\frac{\ddot{a}}{a}=-\pi G \rho(1+w)(1+3 w)
$$

so that the condition for positive acceleration is $-1<w<-1 / 3$. This is to be contrasted to the standard result, $\frac{\ddot{a}}{a}=-(4 \pi G / 3) \rho(1+3 w)$, requiring only that

\footnotetext{
${ }^{1}$ Ref. [10] has also addressed this question in the context of gravitiy with a time-varying speed of light.
} 
$w<-1 / 3$. In (1.2), as $w$ approaches -1 , the acceleration would disappear, coming into conflict with the observations. It must be emphasized however that since the connection between $w$ and acceleration is no longer the same when eq. (1.1) is adopted, one should the modified expansion law.

In the following we will present results for exponential quintessence potentials, since these proved to be the most promising for overcoming the horizon problem. As will be shown, the modified Friedmann equation enlarges the range of potential parameters which avoid a future horizon, but there is also an allowed range using the normal Friedmann equation. In the penultimate section it will be argued that these solutions do not require any more fine tuning than those arising from other models of quintessence.

\section{Quintessence evolution and event horizon}

The quintessence field $Q$ with potential $V$ has the usual equation of motion, $\ddot{Q}+$ $3 H \dot{Q}+\frac{d V}{d Q}=0$. Its pressure and energy density are given by $\rho_{Q}=\frac{1}{2} \dot{Q}^{2}+V$ and $p_{Q}=\frac{1}{2} \dot{Q}^{2}-V$. To explore cosmology both in the usual case and with the modified Friedmann equation (1.1) we will introduce a parameter $x=0,1$ and write the Hubble rate as

$$
H^{2}=\kappa_{x}^{2}\left(\left(1+\frac{x}{3}\right) \rho_{r}+\rho_{m}+\frac{1}{2}(1+x) \dot{Q}^{2}+(1-x) V\right)
$$

where $\kappa_{x}^{2}=8 \pi G /\left(1+\frac{x}{3}\right)$ and $\rho_{r}$ and $\rho_{m}$ are the radiation and matter energy densities, respectively. It thus reduces to the standard equation when $x=0$ and the self-tuning one (1.1) when $x=1$. Guided by the CMB data, we assume the curvature term in $H^{2}$ to be absent.

Rather than integrating with respect to time $t$, it is convenient to think in terms of redshift, $1+z=1 / a(t)$, where we take the present scale factor $a\left(t_{0}\right)$ to be unity. Further defining $u=\ln (1+z)$, and rescaling the fields and energy densities via

$$
\begin{array}{ll}
\hat{Q}=\kappa_{x} Q, & \hat{\rho}_{i}=\kappa_{x}^{2} H_{0}^{-2} \rho_{i}, \\
\hat{V}=\kappa_{x}^{2} H_{0}^{-2} V, & \hat{H}=H / H_{0},
\end{array}
$$

the quintessence equation of motion and Friedmann equation can be written in the dimensionless form

$$
\begin{aligned}
\hat{Q}^{\prime \prime} & =\hat{H}^{-2}\left[\left(\left(1+\frac{x}{3}\right) \hat{\rho}_{r}+\frac{3}{2} \hat{\rho}_{m}+(1-x) \hat{V}\right) \hat{Q}^{\prime}-\left(1-x \hat{Q}^{\prime 2}\right) \frac{\partial \hat{V}}{\partial \hat{Q}}\right] \\
\hat{H}^{2} & =\frac{\left(1+\frac{x}{3}\right) \hat{\rho}_{r}+\hat{\rho}_{m}+(1-x) \hat{V}}{1-\frac{1}{2}(1+x) \hat{Q}^{\prime 2}},
\end{aligned}
$$

where primes denote $\frac{d}{d u}$. The matter and radiation densities scale with $u$ like $\hat{\rho}_{m}=$ $\hat{\rho}_{m, 0} e^{3 u}$ and $\hat{\rho}_{r}=\hat{\rho}_{r, 0} e^{4 u}$ with respect to their present values at $u=0$. These 
dimensionless equations are well-suited to numerical integration, which is the main technique of our investigation. Notice that conventional time $t$ has been eliminated, and $u=\ln (1+z)=-\ln (a)$ now plays the role of the time variable.

In comparing the properties of the quintessence field to observations, we will refer to the fractions of the critical energy density, and the equation of state. The former are defined as

$$
\begin{aligned}
\Omega_{i} & =\frac{\kappa_{x}^{2} \rho_{i}}{H^{2}}=\frac{\hat{\rho}_{i}}{\hat{H}^{2}} \\
\Omega_{Q} & =\frac{\kappa_{x}^{2}}{H^{2}}\left(\frac{1+x}{2} \dot{Q}^{2}+(1-x) V\right)=\frac{1+x}{2} \hat{Q}^{\prime 2}+\frac{1}{\hat{H}^{2}}(1-x) \hat{V},
\end{aligned}
$$

which satisfy $\Omega_{r}+\Omega_{m}+\Omega_{Q}=1$. As for the quintessence equation of state, it is given by

$$
w=\frac{\dot{Q}^{2}-2 V}{\dot{Q}^{2}+2 V}=\frac{\hat{H}^{2} \hat{Q}^{\prime 2}-2 \hat{V}}{\hat{H}^{2} \hat{Q}^{\prime 2}+2 \hat{V}}
$$

This follows from the fact that $\dot{Q}=-H Q^{\prime}$.

In standard cosmology, $w<-1 / 3$ is the criterion for acceleration. But as noted in the introduction, the relation between $w$ and acceleration is modified for the selftuning scenario with $x=1$. It is therefore useful to have another quantity indicative of acceleration, which can be more directly related to the observations of high- $z$ supernovae. Let us first review what is actually constrained [14]: it is the distance modulus ( $m-M=$ apparent minus absolute magnitude) of the SN versus its redshift, where $m-M=5 \log _{10}\left(d_{L} / \mathrm{Mpc}\right)+25$, and the luminosity distance $d_{L}(z)$ is given by $(1+z) H_{0} \Delta(z)$, with

$$
\Delta(z)=\int_{t(z)}^{t_{0}} \frac{d t}{a(t)}=\int_{0}^{z} \frac{d z^{\prime}}{\hat{H}\left(z^{\prime}\right)}=\int_{0}^{\ln (1+z)} \frac{e^{u}}{\hat{H}(u)} d u
$$

In a flat universe with only matter and cosmological constant components, $\Omega_{m}+\Omega_{\Lambda}=$ 1 , one would have

$$
\Delta_{\Lambda} \equiv \int_{1}^{1+z} \frac{d x}{\left(x^{3} \Omega_{m}+\Omega_{\Lambda}\right)^{1 / 2}}
$$

The high- $z$ SN results essentially try to measure $\Delta(z)$ as a function of $z$ in order to fit $\Omega_{\Lambda}$. We therefore define a phenomenological parameter, $\Omega_{\Lambda}^{\mathrm{eff}}$ :

$$
\Omega_{\Lambda}^{\mathrm{eff}} \equiv \Omega_{\Lambda} \text { such that } \Delta_{\Lambda}(3 / 4)=\Delta(3 / 4)
$$

i.e., $\Omega_{\Lambda}^{\mathrm{eff}}$ is the amount of $\Omega_{\Lambda}$ for pure cosmological constant that would be required to give the same value of $\Delta(z)$ as is produced by the quintessence model. This is a $z$-dependent definition, and we somewhat arbitrarily take the value $z=0.75$ because this is roughly the redshift where the current SN data are which are the most sensitive to $\Omega_{\Lambda}$. 
The function $\Delta$ is also relevant for determining whether an event horizon appears in the spacetime, since the coordinate distance traveled by a photon between the present and a future (negative) value of $z$ is precisely $\Delta(z)$. The criterion for an event horizon is that $\lim _{z \rightarrow-1} \Delta(z)<\infty$. In this case the photon travels a finite coordinate distance in an infinite time, and this determines the position of the horizon for the observer that emitted the photon: no signals originating from beyond that position will ever be able to reach him. For ease of representation, we will define the following measure of horizon formation:

$$
D \equiv \lim _{a \rightarrow \infty} \frac{\partial \ln \Delta}{\partial \ln a}= \begin{cases}0, & a(t) \sim e^{H t}, \text { horizon exists } \\ \frac{1}{q}-1, & a(t) \sim t^{q}, \text { no horizon if } q<1\end{cases}
$$

Here $q=\frac{2}{3(1+w)}$ if the dominant component has equation of state $p=w \rho$. For example, $D=1 / 2$ in a universe which behaves as though it is matter dominated $(q=2 / 3)$ at very late times. We will see below that $D=1 / 2$ is the maximum value that arises in the quintessence models which we consider.

\section{Results}

We have examined some of the popular choices of the quintessence potential, $V(Q)$, including inverse powers, exponentials, and combinations of the two. For avoiding the event horizon, the exponential potential (first considered in [15], and subsequently in [16]-18]) seems most promising:

$$
\hat{V}(\hat{Q})=\hat{V}_{0} e^{-\beta \hat{Q}}
$$

This potential has only a single free parameter, $\beta,^{2}$ once the constraint that $\Omega_{Q}=$ $1-\Omega_{m}$ is imposed for the present epoch, for this determines $V_{0}$. The statement is strictly true if one assumes that initially $\hat{Q}^{\prime}=0$, since the initial value of $\hat{Q}$ itself can be absorbed into the definition of $\hat{V}_{0}$. However, even if $\hat{Q}^{\prime} \neq 0$ initially, the quintessence field converges to an attractor solution [16]-18], as shown in figure 1): the two solutions corresponding to different initial conditions converge to the same functional form after some time. In this example, we numerically integrated the equation of motion for the case $\Omega_{m}=0.25$ starting from initial conditions at $u=\ln (1+z)=12\left(z=1.6 \times 10^{5}\right)$, during the radiation dominated era with initial conditions $\hat{Q}=0^{3}$ and $\hat{Q}^{\prime}=0$ or 1 . Although the early behavior of $\hat{Q}$ is clearly affected by the difference in initial $\hat{Q}^{\prime}$, both solutions join their common trajectory well before quintessence starts to dominate in the present era. Figure 2 shows the corresponding quintessence equation of state, $w$, in the two cases. In this example it

\footnotetext{
${ }^{2}$ in the notation of ref. [16], $\beta=\sqrt{6} a$, and in that of refs. [17, 18], $\beta=\sqrt{3} \lambda$, assuming the normal Friedmann equation.

${ }^{3}$ The initial condition on $\hat{Q}$ is not significant since it can be absorbed into $\hat{V}_{0}$.
} 
is clear that quintessence can contribute to the acceleration during the period when $w=-1$, whereas $w \rightarrow-0.25$ in the future, which is larger than $-1 / 3$ and therefore cannot cause acceleration.

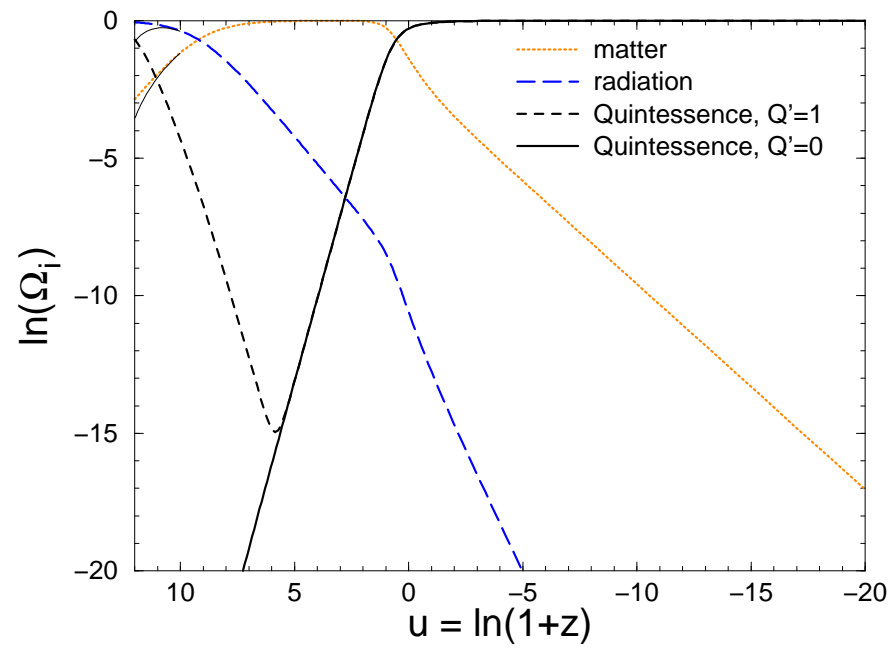

Figure 1: $\Omega_{r}, \Omega_{m}$ and $\Omega_{Q}$ as a function of $u=\ln (1+z)$ for the potential $\hat{V}=\hat{V}_{0} e^{-2.6 \hat{Q}}$ (i.e., $\beta=2.6$ ). The initial conditions at $u=12$ are $\hat{Q}^{\prime}=0$ and $\hat{Q}^{\prime}=1$, respectively.

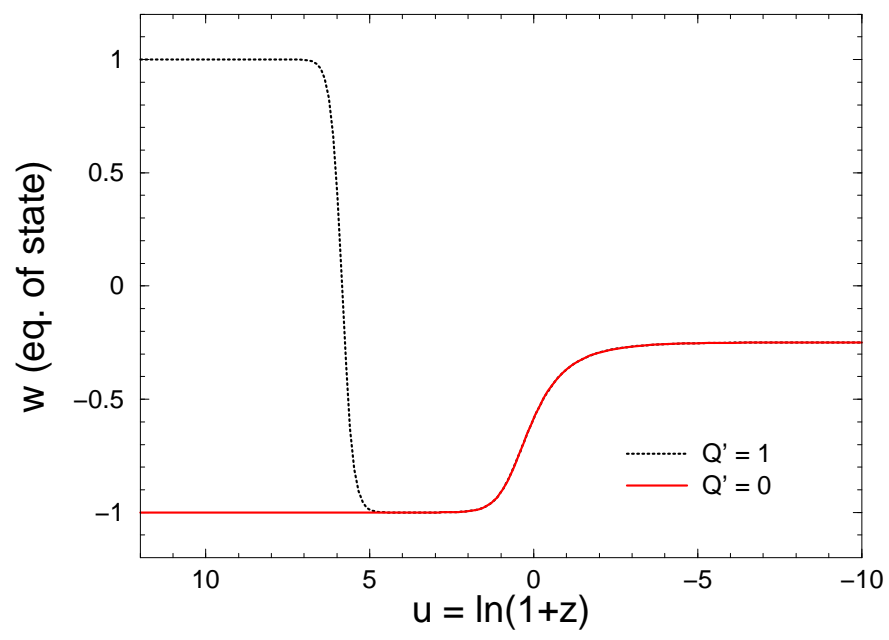

Figure 2: The quintessence equation of state, $w$, as a function of $u$, for the same two sets of initial conditions as in figure 1 .

Now let us consider the fate of the universe at very late times, for the interesting range of potential parameters. We find that a range of $\beta$ values exists such that the cosmological expansion is accelerating today even though at late times it will revert to a power law, $a \sim t^{q}$, with $q \leq 2 / 3$. This is illustrated by the solid curves of figure 3, which again were made assuming that $\Omega_{m}=0.25$ and the initial conditions $\hat{Q}=\hat{Q}^{\prime}=0$ at $u=12$. From the middle curve, the horizon formation parameter $D$ (eq. (2.11)), we see that an event horizon is avoided if $\beta \gtrsim 2.4$. It is interesting to 
note that for larger values of $\beta, D \rightarrow 1 / 2$, just as it would for a matter-dominated universe, even though quintessence is dominating at late times. This happens because the solutions have the property that $\rho_{Q} \sim a^{-3}$, just as though the universe was matter-dominated [17].

However, very large values of $\beta$ do not yield sufficient acceleration at present times to be consistent with observations. This can be seen from the top and bottom curves, showing $\Omega_{\Lambda}^{\mathrm{eff}}$ (defined in eq. (2.10)) and $w$ (eq. (2.7)) respectively. The $99 \%$ confidence level SN limits are $w<-0.5$ for $\Omega_{m}=0.25$ and $\Omega_{\Lambda}^{\text {eff }}>0.5$ for a flat universe. It is difficult to apply the limit on $w$ in a quantitative way in the present model because of the fact that $w$ is changing very rapidly between the redshifts of $z=1 / 2$, where much of the SN data is clustered, and the present, $z=0$. This rapid variation can be seen in the example of figure 2 as well as by comparing the bottom sets of curves in figure 3 . On the other hand, the limit on $\Omega_{\Lambda}^{\text {eff }}$ can be applied in a straightforward way to give $\beta \lesssim 2.8{ }^{4}$ Thus it is possible to satisfy the observational constraints without getting an event horizon if $2.4 \lesssim \beta \lesssim 2.8$.

We have also done the same analysis using the self-tuning Friedmann equation, shown by the dashed curves of figure 3 . One sees that the range of $\beta$ for which no horizon forms is enlarged to $\beta \gtrsim 1.8$. This agrees with the intuitive expectation that acceleration is reduced in this case, compared to the standard Friedmann equation. But at the same time, $\Omega_{\Lambda}^{\mathrm{eff}}$ and $|w|$ are decreased, making it more difficult to obtain the observed acceleration. Since the connection between $\frac{\ddot{a}(t)}{a}$ and $w$ is no longer the same as assumed in the analysis of the SN data, we again take advantage of the $\Omega_{\Lambda}^{\text {eff }}$ parameter. Demanding that $\Omega_{\Lambda}^{\mathrm{eff}}>0.5$ gives $\beta<2.4$. The allowed range consistent with no horizon, $1.8 \lesssim \beta \lesssim 2.4$, is thus shifted and slightly widened relative to the normal case.

It is easy to elucidate the origin of our loophole to the horizon-formation arguments put forward in references [7]-9]. These analyses assume that quintessence is dominating very strongly in the present, or equivalently that its equation of state does not change from its present value. However this approximation is not valid for the solutions we have presented, such as in figure 1. In the present epoch, $u=0$, the equation of state $w$ for these solutions is always going through a transition from -1 to some value greater than $-1 / 3$, so as to avoid the horizon. In the case of solutions with small $\beta<2.4$, it is also true that $w$ changes near $u=0$, but its final value at large times is $w<-1 / 3$, leading to a horizon.

\footnotetext{
${ }^{4}$ Naively one might expect that $\Omega_{\Lambda}^{\text {eff }}=1-\Omega_{m}$ should be satisfied, which would rule out all values of $\beta$ shown, but we remind the reader that $\Omega_{\Lambda}^{\mathrm{eff}}$ is defined as the amount of real $\Omega_{\Lambda}$ that would give the same amount of current acceleration as the given quintessence model. Since quintessence is less efficient at causing acceleration than is vacuum energy, it is not surprising that $\Omega_{\Lambda}^{\text {eff }}$ is less than $\Omega_{Q}$.
} 


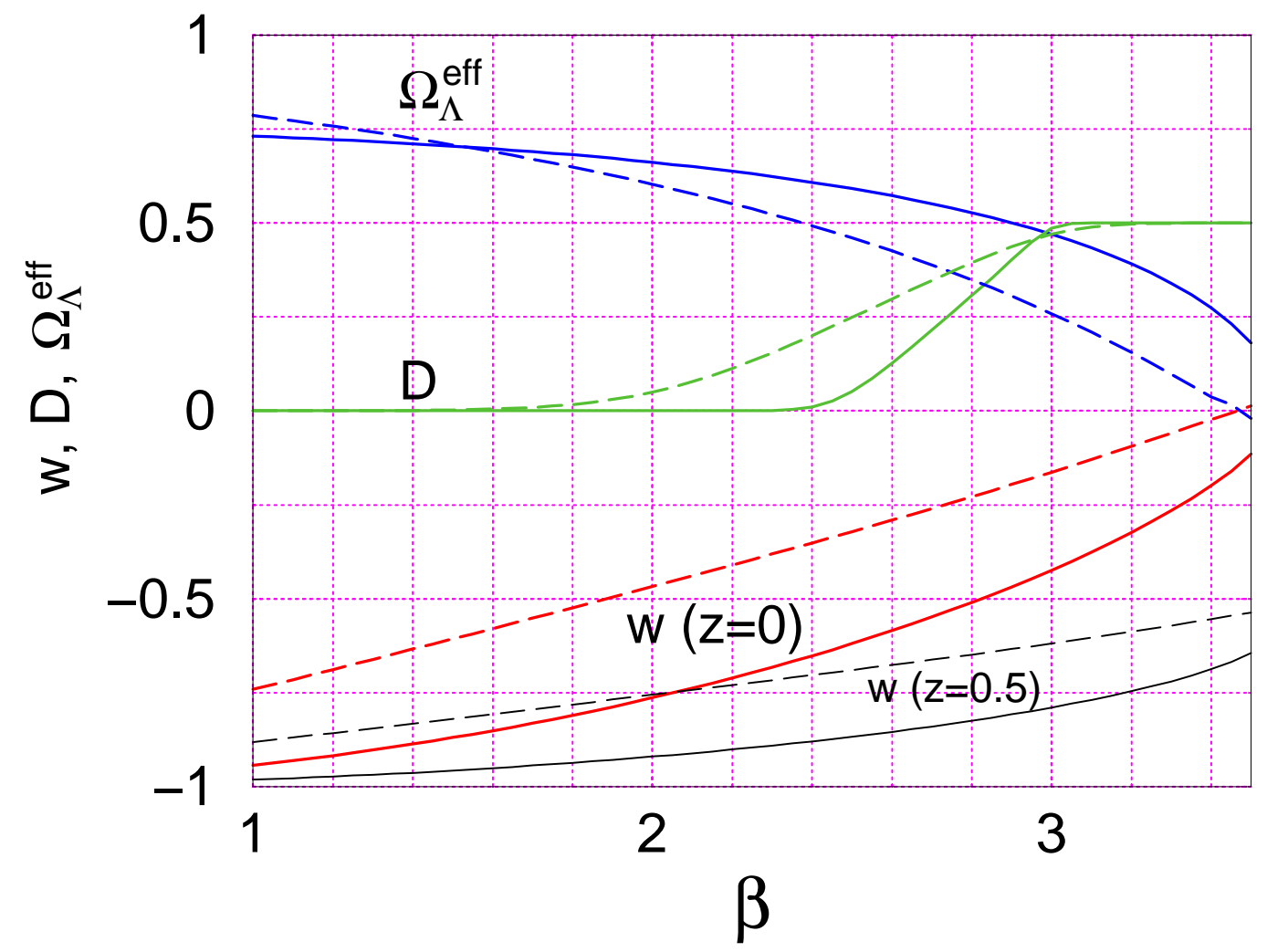

Figure 3: From bottom to top: the quintessence equation of state $w$, at redshifts $z=1 / 2$ and at $z=0$, the horizon formation parameter $D$ (eq. (2.11)), and the SN effective $\Omega_{\Lambda}$, $\Omega_{\Lambda}^{\mathrm{eff}}$ (eq. (2.10)), as a function of the potential parameter $\beta$. Solid lines are for the normal Friedmann equation $(x=0)$, dashed are for the self-tuning one $(x=1)$.

\section{Naturalness}

In this section we discuss the question of how much fine tuning is needed to obtain the desired solutions. The exponential potential has been somewhat disparaged because of the emphasis on solutions which reach the scaling regime, where $w$ is constant, very early in the evolution. Such solutions are uninteresting in light of the current data because they maintain a constant value of $\Omega_{Q}$. Since $\Omega_{Q}$ must be less than about $15 \%$ at nucleosynthesis or during large scale structure formation, this would render its contribution too small to account for the present acceleration. But as pointed out in [19], this negative conclusion can be circumvented by assuming that quintessence is far from the late-time attractor solution in the not-too-distant past, so that $w$ can evolve, which is exactly the situation for the solutions presented here.

Does the fact that these quintessence solutions start out far from the late-time attractors make them less natural? We argue that this is not the case. All quintessence models require one tuning in order to achieve $\Omega_{Q}=1-\Omega_{m}$ today, and this is the only one which we have invoked. The tuning is imposed as a particular value of the 
combination

$$
\tilde{V}_{0} \equiv V_{0} e^{-\beta \hat{Q}_{i}}
$$

where $\hat{Q}_{i}$ is the initial value of $\hat{Q}$. The value of $\tilde{V}_{0}$ required to make $\Omega_{Q}=1-\Omega_{m}$ today depends on the initial velocity, or equivalently $\hat{Q}_{i}^{\prime}$. In the very early universe the kinetic energy of quintessence typically dominates over its potential energy unless $\hat{Q}_{i}^{\prime}$ is exactly zero (see eq. (2.6)), so this amounts to a choice for the initial value of $\Omega_{Q, i} \cong(1+x) \hat{Q}_{i}^{\prime 2} / 2$. Figure 1 1 shows that whether $\Omega_{Q, i} \cong 0$ or 1 , the recent evolution of the quintessence is identical, so long as $\tilde{V}_{0}$ takes the right value. In this sense, we can say that the models under consideration are very insensitive to the initial conditions. This conclusion in no way depends on our choice for the initial time. Figure 4 shows the evolution of $\Omega_{i}$ for the same parameters as in fig. 1, except now the initial redshift is taken to be $10^{17}$, corresponding to an initial temperature of 100 $\mathrm{TeV}$. Not only is the late-time evolution unaffected, but the choice of $V_{0}$ is identical if $\hat{Q}_{i}^{\prime}=0$, and $V_{0}$ only changes by a factor of 5 relative to the later initial condition if $\hat{Q}_{i}^{\prime} \neq 0$.

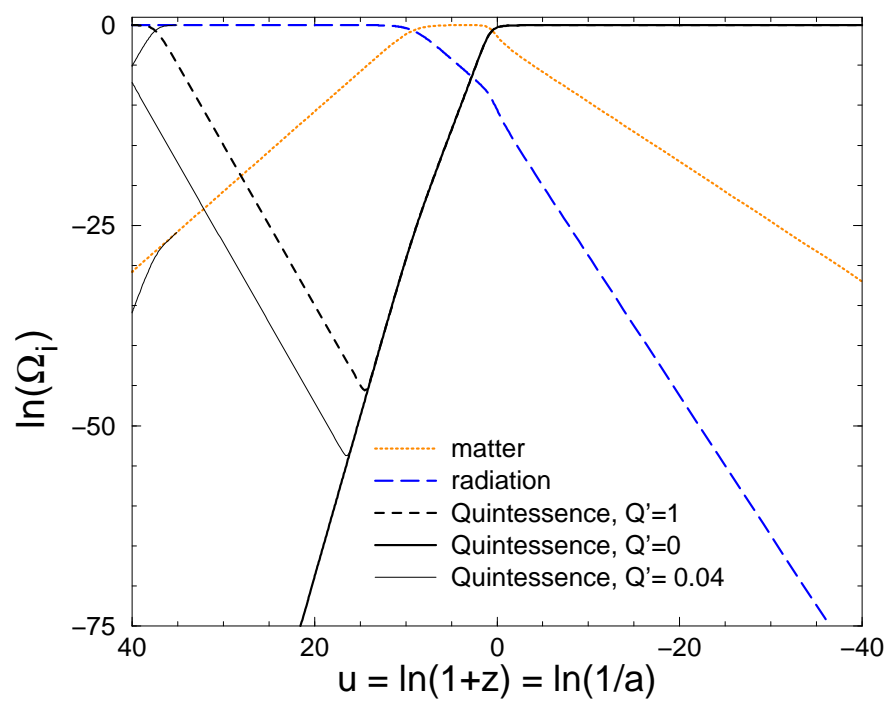

Figure 4: Same as fig. 1, except starting at redshift $z=10^{17}$ instead of $z=10^{5}$ (and the additional initial condition $\hat{Q}_{i}^{\prime}=0.04$ is also shown, which corresponds to inflationary initial conditions with equipartition of energy). This demonstrates the insensitivity of the solution to the choice of the initial time.

Another aspect of naturalness is the value of $\tilde{V}_{0}$ required. If $\hat{Q}_{i}^{\prime} \ll 1$ (hence $\Omega_{Q, i} \ll 1$ ), as would be natural in inflation if equipartition was realized [17, 19], then $\tilde{V}_{0}$ must be of order the present critical density, $\tilde{V}_{0} \sim\left(10^{-3} \mathrm{eV}\right)^{4}$. (The evolution with such an initial condition, with $\Omega_{Q, i}=10^{-3}$, hence $\hat{Q}_{i}^{\prime}=0.04$, is illustrated in figure 4.) This looks unnaturally small in particle physics units, but one advantage of the exponential potential is that the smallness of $\tilde{V}_{0}$ can be explained by a moderately large value of $\hat{Q}_{i}$. For example, if $V_{0} \sim(\mathrm{TeV})^{4}$, then $Q_{i}$ should be of order $50 M_{p}$, which is not such a disturbing hierarchy. 
The final aspect of naturalness is how sensitive the solution is to small changes in $V_{0}$. Changing $V_{0}$ causes a shift in the time when the quintessence and matter energy densities become comparable, which is related to the so-called coincidence problem: why is it that quintessence is just starting to dominate in the present epoch? It can be shown that scaling the potential by a factor of $V_{\text {new }} / V_{0}$ leads to the following dependence in the redshift of matter-quintessence equality, $z_{m-q}$ :

$$
z_{m-q}=0.71+\left(\frac{V_{\text {new }}}{V_{0}}\right)^{1 / 3} .
$$

The power $1 / 3$ is just coming from the fact that $V_{0}$ must be of order the critical density at $z_{m-q}$, and density scales with redshift like $(1+z)^{3}$. Therefore, in some sense the value of $z_{m-q}$ is rather insensitive to the value of $V_{0}$; the coincidence problem would be much worse if the dependence was through a higher power.

\section{Conclusion}

We have shown that it is possible to evade the cosmological event horizon which might pose a difficulty for deriving quintessence from string theory: for a range of $2.4 \lesssim \beta \lesssim 2.8$ with the conventional Friedmann equation, or $1.8 \lesssim \beta \lesssim 2.4$ for the self-tuning variant, the exponential potential $V(Q)=V_{0} e^{-\beta \kappa_{x} Q}$ gives this outcome. We also tried other kinds of potentials, such as inverse powers, $V \sim Q^{-p}$, but for these the development of a future horizon was found to be inevitable.

We began this work with the idea that a self-tuning Friedmann equation might make it easier to avoid a cosmological horizon in a quintessential universe. The outcome is that self-tuning does not really make a big difference: eternal acceleration can be avoided with or without self-tuning. Of course self-tuning is still very interesting, because it allows us to work with a larger class of potentials,

$$
V=V_{1}+V_{0} e^{-\beta \hat{Q}}
$$

since only in the self-tuning case is the evolution completely insensitive to the value of $V_{1}$. It is likely that there are other problems with self-tuning, since the strength of gravity on subgalactic scales is known to be consistent with the normal Planck mass, whereas gravity looks effectively weaker on cosmological scales in the selftuning case. This is suggestive of the presence of an extra scalar component like a massive Brans-Dicke field, whose limited range accounts for the difference between the effective Planck mass at large and small distance scales. However the couplings of such a field to matter are very highly constrained by precision tests of general relativity in the solar system, like the precession of the perihelion of Mercury. ${ }^{5}$

\footnotetext{
${ }^{5}$ I thank Maxim Pospelov for discussions on this point.
} 
Regardless of self-tuning however, the class of solutions we have discussed seem sufficiently natural to warrant consideration as a strong candidate for the dark energy which is presently observed. It is expected that progress in the observations of high- $z$ supernovae (the SNAP experiment [20]) will soon be able to distinguish this kind of model from others through an accurate determination of the time dependence of the equation of state [21]. The very large present time-dependence of $w$ in the exponential models makes them particularly interesting in this respect. Figure 5 shows $d w / d z$ at redshift $z=0.5$ for the relevant range of $\beta$. Thus if the SNAP experiment was to measure that $d w / d z<-0.26$ at $z=0.5$, it would indicate that the universe will stop accelerating in the future and thus avoid an event horizon, in the context of the model discussed here.

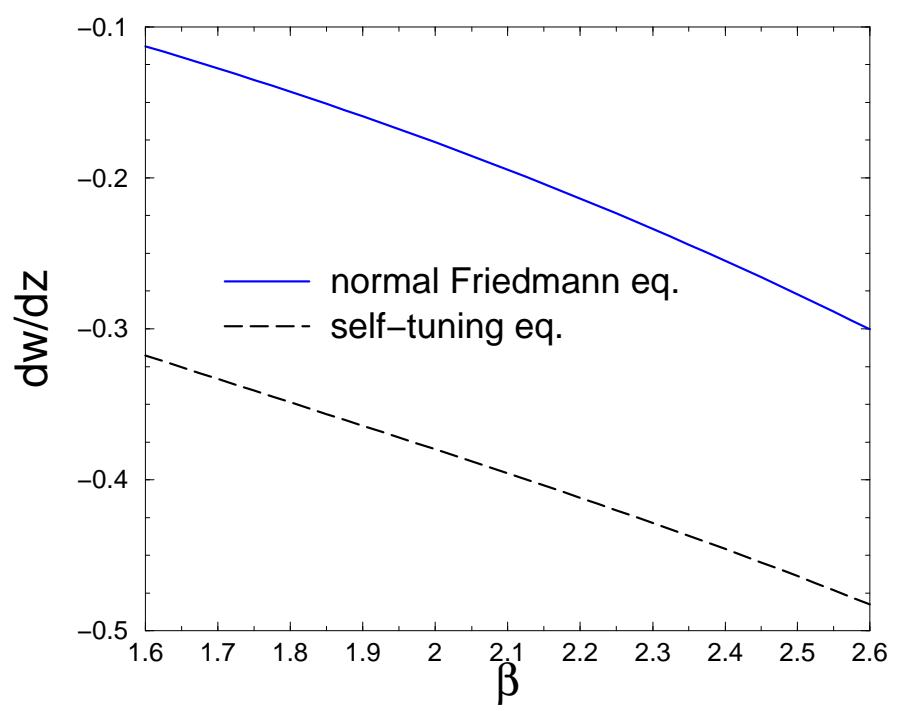

Figure 5: Rate of change of the quintessence equation of state, $d w / d z$, at $z=1 / 2$ as a function of the potential parameter $\beta$. In the region of $\beta=2.4, d w / d z \approx 0.23-0.2 \beta$ for the normal Friedmann equation, and near $\beta=1.8, d w / d z \approx-0.07-0.16 \beta$ for the self-tuning one.

Note added: as this work was being finished, ref. [22] appeared, which presents a different quintessence model that also avoids the future horizon.

\section{Acknowledgments}

I thank the theory group of Lawrence Berkeley Laboratory for their hospitality while this work was being finished.

\section{References}

[1] S. Dodelson and L. Knox, Phys. Rev. Lett. 84, 3523 (2000) astro-ph/9909454;

L. M. Griffiths, A. Melchiorri and J. Silk, astro-ph/0101413. 
[2] J. R. Primack, Nucl. Phys. Proc. Suppl. 87, 3 (2000); astro-ph/0007187.

[3] G.F. Smoot, talk given at DOE Program Review, LBL, 22 May 2001

[4] S. Perlmutter et al. [Supernova Cosmology Project Collaboration], Astrophys. J. 517, 565 (1999) astro-ph/9812133.

[5] R. R. Caldwell, R. Dave and P. J. Steinhardt, Phys. Rev. Lett. 80, 1582 (1998) astro-ph/9708069.

[6] T. Banks, hep-th/0007146;

T. Banks and W. Fischler, hep-th/0102077.

[7] W. Fischler, A. Kashani-Poor, R. McNees and S. Paban, hep-th/0104181.

[8] S. Hellerman, N. Kaloper and L. Susskind, hep-th/0104180.

[9] X. He, astro-ph/0105005.

[10] J. W. Moffat, hep-th/0105017.

[11] N. Arkani-Hamed, S. Dimopoulos, N. Kaloper and R. Sundrum, Phys. Lett. B 480, 193 (2000) hep-th/0001197;

S. Kachru, M. Schulz and E. Silverstein, Phys. Rev. D 62, 045021 (2000) hepth/0001206];

C. Csáki, J. Erlich, C. Grojean and T. Hollowood, Nucl. Phys. B 584, 359 (2000) hep-th/0004133.

[12] S. Förste, Z. Lalak, S. Lavignac and H. P. Nilles, Phys. Lett. B481, 360 (2000) hepth/0002164;

P. Binetruy, J. M. Cline and C. Grojean, Phys. Lett. B 489, 403 (2000) hepth/0007029.

[13] S. M. Carroll and L. Mersini, hep-th/0105007.

[14] M. J. White, Astrophys. J. 506, 495 (1998) astro-ph/9802295.

[15] B. Ratra and P. J. Peebles, Phys. Rev. D 37, 3406 (1988).

[16] C. Wetterich, Astron. Astrophys. 301, 321995 [hep-th/9408025].

[17] P. G. Ferreira and M. Joyce, Phys. Rev. Lett. 79, 4740 (1997) astro-ph/9707286;

Phys. Rev. D 58, 023503 (1998) astro-ph/9711102.

[18] E.J. Copeland, A.R. Liddle and D. Wands, Phys. Rev. D57, 4686 (1998) grqc/9711068].

[19] I. Zlatev, L. Wang and P. J. Steinhardt, Phys. Rev. Lett. 82, 896 (1999) astro$\mathrm{ph} / 9807002]$

P. J. Steinhardt, L. Wang and I. Zlatev, Phys. Rev. D 59, 123504 (1999) astroph/9812313. 
[20] P. Nugent [SNAP Collaboration], "SNAP: Supernova / acceleration probe: An experiment to measure the properties of the accelerating universe,". presented at 2nd Tropical Workshop On Particle Physics And Cosmology, 1-6 May 2000, San Juan, Puerto Rico; snap.lbl.gov.

[21] I. Maor, R. Brustein and P. J. Steinhardt, Phys. Rev. Lett. 86, 6 (2001) astro$\mathrm{ph} / 0007297]$;

T. Chiba and T. Nakamura, Phys. Rev. D 62, 121301 (2000) astro-ph/0008175;

J. Weller and A. Albrecht, Phys. Rev. Lett. 86, 1939 (2001) astro-ph/0008314;

V. Barger and D. Marfatia, Phys. Lett. B 498, 67 (2001) astro-ph/0009256;

D. Huterer and M. S. Turner, astro-ph/0012510

[22] E. Halyo, hep-ph/0105216. 